Ann. Génét. Sél. anim., r980, 12 (4), 395-400.

\title{
Observations chromosomiques chez le sanglier français (Sus scrofa scrofa)
}

\author{
C. P. POPESCU (*), J. P. QUÉRÉ (**) et P. FRANCESCHI (*)
}

avec la collaboration technique de Jeannine Boscher (*)

(*) Laboratoire de Cytogénétique, C.N.R.Z., I.N.R.A., 78350 Jouy-en-Josas, France

(**) Laboratoire de la Faune Sauvage, C.N.R.Z., I.N.R.A., 78350 Jouy-en-Josas, France

\begin{abstract}
Résumé
Une étude cytogénétique a été entreprise sur un lot de sangliers Français Continentaux et Corses et des hybrides sanglier $\times$ porc domestique. Les sangliers Continentaux ont révélé un caryotype à 36 chromosomes, à l'exception d'un seul animal provenant d'un élevage et qui possédait 38 chromosomes. Les sangliers Corses présentaient 38 chromosomes identiques à ceux du porc domestique. L'hypothèse d'une origine du sanglier Corse par marronnisation d'une souche domestique a été avancée.
\end{abstract}

Les travaux de ces dernières années, et en particulier ceux de Trknonov et al. (1974, I975), ont mis en évidence un polymorphisme chromosomique chez le sanglier. Ainsi, la sous-espèce d'Europe Occidentale (Sus scrofa scrofa) possède une garniture chromosomique de $2 n=36$, alors que les sangliers d'Asie Centrale (Sus scrofa ferus, Sus scrofa attila, Sus scrofa nigripes) et d'Extrême-Orient (Sus scrofa ussuricus) ont 36,37 ou 38 chromosomes. Certaines populations de sangliers d'Europe de 1'Est possèdent, elles aussi, 38 chromosomes (Zivkovic et al., 197I) ainsi que la sous-espèce Sus scrofa leucomystax de l'archipel japonais (MURAмото et al., I965). Les porcs domestiques (Sus scrofa domesticus), examinés à ce jour dans différentes régions du monde et appartenant à des races aussi diverses que le Landrace français ou la Mei-chan chinoise (POPESCU, non publié), ont toujours présenté 38 chromosomes.

Ces derniers temps, le nombre d'élevage de sangliers s'est considérablement accru en France (MAUGE'T et al., r977) et il nous a paru important de préciser le caryotype du sanglier français afin de pouvoir déceler, à l'aide du critère chromosomique, d'éventuels croisements avec le porc domestique. Cette note a pour but de présenter les résultats d'une étude cytogénétique effectuée sur des sangliers continentaux et corses, purs et croisés avec des porcs domestiques (tabl. I).

L'étude cytogénétique a été réalisée sur des cultures de sang effectuées selon la méthode de DE GRouChy et al. (I964). Les chromosomes ont été examinés sur des préparations colorées au Giemsa et en marquage $R$, après incorporation de 
TABLEAU I

Origines effectifs, phénotypes et caryotypes des animaux étudiés

Origin, numbers, phenotypes and caryotypes of the stuaied animals

\begin{tabular}{|c|c|c|c|c|}
\hline 昜 & Origine & $\begin{array}{l}\text { Nombre } \\
\text { d'animaux } \\
\text { étudiés }\end{array}$ & Phénotype coloré & Caryotype \\
\hline \multirow{4}{*}{ 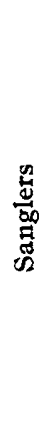 } & Chambord, parc clos de murs & $\begin{array}{r}25 \\
I\end{array}$ & $\begin{array}{l}\text { Coloration sauvage normale } \\
\text { Coloration claire }\end{array}$ & $\begin{array}{l}36 \\
36\end{array}$ \\
\hline & Arc-en-Barrois, forêt ouverte & 3 & Coloration sauvage normale & 36 \\
\hline & 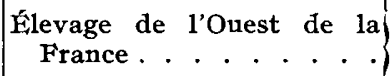 & $\begin{array}{l}6 \\
I\end{array}$ & $\begin{array}{l}\text { Coloration sauvage normale } \\
\text { Roux }\end{array}$ & $\begin{array}{l}3^{6} \\
3^{8}\end{array}$ \\
\hline & Corse, forêt ouverte. & $\begin{array}{l}6 \\
\mathrm{r}\end{array}$ & $\begin{array}{l}\text { Coloration sauvage } \\
\text { Noir }\end{array}$ & $\begin{array}{l}38 \\
38\end{array}$ \\
\hline \multirow{2}{*}{$\frac{\mathscr{d}}{\mathscr{W}}$} & 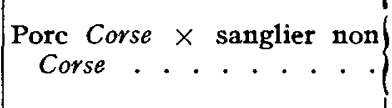 & $\begin{array}{l}7 \\
7 \\
5\end{array}$ & $\begin{array}{l}\text { Coloration sauvage } \\
\text { Noir, tête blanche } \\
\text { Noir, poil frisé }\end{array}$ & $\begin{array}{l}37 \\
37 \\
37\end{array}$ \\
\hline & Porc Corse $\times$ sanglier Corse & I 7 & $\left\{\begin{array}{l}\text { Différentes colorations (blanc } \\
\text { taches noires, face blanche, } \\
\text { rousse) }\end{array}\right.$ & $3^{8}$ \\
\hline
\end{tabular}

BUDR, selon une méthode utilisée pour les chromosomes des bovins (Popescu, I975).

Les 28 sangliers à coloration sauvage normale, prélevés lors de chasses dans le Parc de Chambord et la forêt d'Arc-en-Barrois, présentent tous un caryotype à 36 chromosomes (fig. $\mathbf{x} b$ ). Le seul sanglier à coloration claire prélevé à Chambord présente, lui aussi, un caryotype à 36 chromosomes tout à fait comparable à celui des sangliers à phénotype normal. Ceci corrobore avec les résultats obtenus par MAUGeT et al. (I977) sur une population de sangliers à robe claire de la forêt de Chizé et par JoTTERAND-BELIOMO et BAETtig (I980, sous presse) sur 2 sangliers de couleur claire en Suisse.

Sur les 7 animaux prélevés dans un élevage, 6 présentaient un caryotype à 36 chromosomes et $\mathrm{I}$ animal à coloration noire avait 38 chromosomes.

Les 7 sangliers Corses et les 17 hybrides des porc Corse $\times$ sanglier Corse étudiés présentaient 38 chromosomes (fig. $2 b$ ) en tous points identiques à ceux du porc domestique (fig. I a). Les hybrides porc Corse $\times$ sanglier non Corse, eux, présentaient 37 chromosomes (fig. $2 a$ ).

La différence entre le caryotype à 36 chromosomes du sanglier continental et celui à 38 chromosomes du sanglier Corse et du porc domestique n'affecte pas le nombre des bras ou Nombre Fondamental (N.F., MATTHEY, I947). Elle provient 

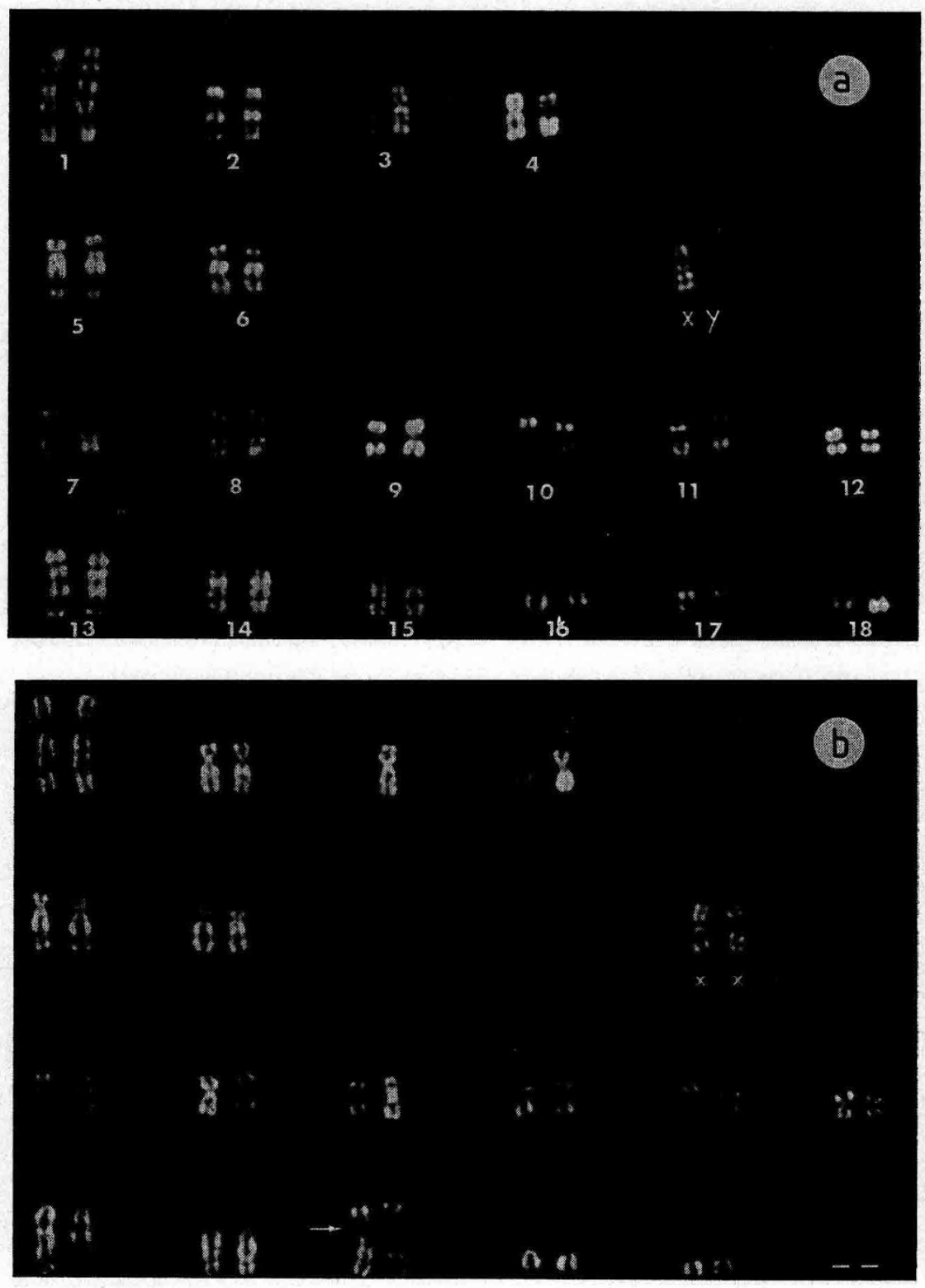

Fig. I $a$ : Caryotype de porc domestique mâle en bandes $R, 2 n=3^{8}$.

$\mathrm{I} b$ : Caryotype de sanglier femelle en bandes $R, 2 n=36$. Les chromosomes fusionnés sont marqués par une flèche.

I $a$ : Caryotype of male domestic pig $R$ banding, $2 n=38$.

$\mathbf{I} b$ : Caryotype of female wildboar $R$ banding, $2 n=36$. The fused chromosomes are indicated with an arrow. 

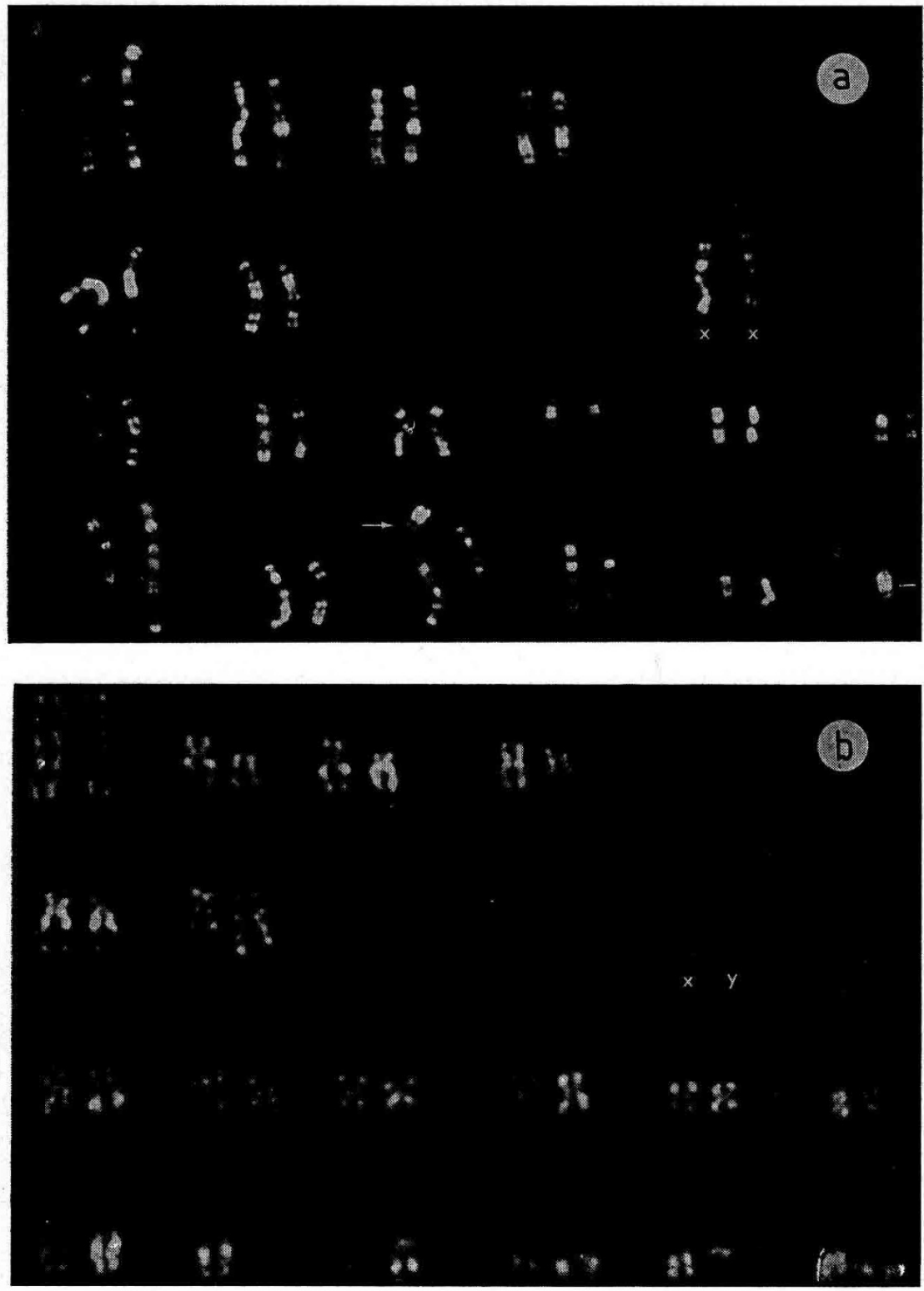

Fig. $2 a$ : Caryotype de croisé porc Corse $\times$ scniglier Continental femelle en bandes $R, 2 n=37$. Le'chromosome fusionné est marqué par une flèche.

$2 b$ : Caryotype de sanglier Corse male en bandes $R, 2 n=38$.

$2 a$ : Caryotype of Corsican pig $\times$ Continental wildboar crossing, $R$ banding, $2 n=37$. The fused chromosome is indicated with an arrow.

$2 b$ : Caryotype of male Corsican wild-boar, $R$ banding, $2 n=38$. 
d'une fusion robertsonienne des paires I5 $_{5}$ et $\mathrm{I} 8$ d'acrocentriques du caryotype à 38 chromosomes qui donne une seule paire de submétacentriques et réduit ainsi le nombre de base de 2 unités. Les hybrides à 37 chromosomes possèdent un seul chromosome fusionné et les deux homologues acrocentriques libres (fig. 2a). On remarque que la fusion trouvée chez le sanglier français implique les paires I5 et I8 d'acrocentriques alors que chez Sus scrofa nigripes, Sus scrofa ussuricus, Sus scrofa attila se sont les paires $\mathrm{I} 6$ et $\mathrm{I}_{4}$ et chez Sus scrofa ferus les paires I5 et I7 (Tiknonov et al., r978). Par conséquence, le polymorphisme chromosomique du sanglier concerne non seulement le nombre chromosomique de base, mais aussi, dans le cas du caryotype à 36 chromosomes, différentes paires d'acrocentriques.

Les résultats confirment le nombre de 36 chromosomes décrit par MaUGET (I977) chez le sanglier français. L'animal de couleur noire présentant un caryotype à 38 chromosomes, originaire d'un élevage, était vraisemblablement un produit de croisement avec le porc domestique. Selon MaUGET, ces produits deviennent après plusieurs générations impossible à distinguer de la forme sauvage.

Ceci indique le danger que représente pour le maintien de l'espèce sauvage pure l'introduction d'animaux domestiques à caryotype différent. En plus, bien que toutes ces sous-espèces à caryotype différent soient inter-fertiles, on ne connaît pas actuellement le comportement du trivalent qui se forme à la méiose chez. les hybrides $F_{1}$ à 37 chromosomes et ses conséquences sur la qualité des gamètes qui en résultent.

En ce qui concerne le sanglier Corse, bien que l'échantillon étudié soit très petit, il pourrait s'agir d'une population à 38 chromosomes. Les données archéologiques indiquant qu'un changement profond de la faune de Mammifères s'est produit en Corse à une époque qui coïncide avec les premiers peuplements de l'île, il n'est pas invraisemblable d'imaginer que l'homme ait joué pour le porc le même rôle actif dans la mutation de la faune insulaire que lui attribue PopLIN (r979) pour le mouflon, en introduisant des sujets domestiques dont certains sont retournés à l'état sauvage. Le sanglier Corse pourrait alors être un autre cas typique de marronnage.

Quant à l'origine du porc domestique, la découverte de plusieurs populations de sangliers à 38 chromosomes rend plausible 1'hypothèse de la domestication à partir d'une de ses formes, sans remaniement chromosomique. L'autre alternative, plus ancienne, la domestication des sangliers à 36 chromosomes, impliquerait une fission centrique transformant une paire de chromosomes submétacentriques en deux paires d'acrocentriques et l'augmentation du nombre de base de deux unités. Or, si la fission centrique n'est pas théoriquement impossible, elle suppose la création d'un nouveau centromère difficilement explicable. D'autre part, la présence de ce polymorphisme produit par différentes fusions centriques semble indiquer ce mécanisme plus fréquent dans l'évolution chromosomique de cette espèce.

$$
\text { Reçu pour publication en janvie } I 98 I \text {. }
$$

\section{Remerciements}

Nous tenons à exprimer nos remerciements à M. G. de ROQUANCourr, commissaire à l'aménagement du Domaine de Chambord pour les facilités de travail qu'il nous a offert et M. D. BouRDON, ingénieur I.N.R.A., pour les prélèvements sanguins. 


\section{Summary \\ Chromosomic observations in french wild boar \\ (Sus scrofa scrofa)}

A cytogenetics study was made in France on a population of Continental and Corsican wild boar out of a lot of hybrids between the wild and domestic pig. All Continental wild-boar living in natural conditions showed $2 n=36$ chromosomes. Only on animal, originating from a private farm, showed $2 n=3^{8}$ chromosomes, identical to the domestic pig chromosomes. On the contrary the Corsican wild boars showed a $2 n=38$ caryotype very similar with the domestic pig caryotype. To explain the origin of Corsican wild-boar the hypothesis of feralization of an early introduced domestic pig is suggested.

\section{Références bibliographiques}

Grouchy J. de, Roubin M., Passage E., 1964. Microtechnique pour l'étude des chromosomes humains à partir d'une culture de leucocytes sanguins. Ann. Génét., 7, 45 .

JotTERAND-BELlomo Martine, BAETtig M., r980. Etude cytogénétique de deux sangliers (Sus scrofa $L$.) de couleur claire, capturés aux environs de Genève (Suisse). (sous presse).

Matrhey R., r948. Les chromosomes des vertébrés. Masson, Paris.

Mauget R., Castet M. C., Maraud R., i977. Étude dynamique et caryotypique d'une population de sangliers à robe claire. C. $R$. Soc. Biol. filiales, 171, 592-596.

Muramoto J., Makion S., Ishikawa T., Kanagawa H., I965. On the chromosomes of the wild-boar and the boar-pig hybrids. Proc. Japan Acad., 41, 236-239.

Popescu C. P., 1975. Essai d'identification des chromosomes bovins (Bos taurus L.) à l'aide

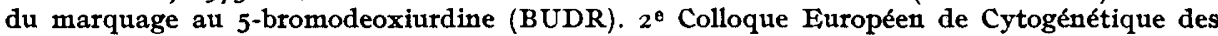
animaux domestiques, Giessen, 29-30 septembre 1975, 59-64.

POPLIN F., r979. Origine du Mouflon de Corse dans une nouvelle perspective, par marronnage. Ann. Génét. Sél. anim., 11, 133-143.

Tikhonov V. N., Troshina A. I., I974. The identification of chromosome rearrangement of the wild and domestic pigs by the Giemsa banding method. Ist World Congress on genetics applied to livestock production. Madrid, Oct. 1974, 3, I93-196.

TikHoNov V. N., Troshina A. I., 1975. Chromosome translocations in the karyotypes of wild-boars Sus scrota $L$. of the european and the asian areas of USSR. Theore. Appl. Genot., 45, 304-308. 